\title{
Evaluación preliminar de patologías debidas a corrosión en una planta industrial.
}

\author{
L. Montani ${ }^{1}$, H. A. Donza ${ }^{1}$ y O. A. Cabrera ${ }^{1 *}$ \\ *Autor de Contacto: ocabrera@,fio.unicen.edu.ar \\ ${ }^{1}$ Facultad de Ingeniería-Universidad Nacional del Centro de la Provincia de Buenos Aires y CIFICEN (UNCPBA- \\ CICPBA-CONICET), Avda. del Valle 5737, 7400, Olavarría, Argentina.
}

\section{RESUMEN}

El presente trabajo se refiere a una evaluación visual preliminar de una planta industrial, con una superficie de $\approx 59000 \mathrm{~m}^{2}$, para determinar el grado de deterioro de sus elementos estructurales pretensados y premoldeados (448 canalones, 7000 losetas y 720 columnas). Se estableció y aplico un índice de daño para cada elemento estructural, en base al cual se propuso el tiempo de intervención para mantener la seguridad y estabilidad de la estructura. Las principales patologías encontradas fueron: eflorescencias y manchas de óxido (10-48\%), fisuras (10-24\%) y desprendimiento del recubrimiento (13-30\%). Los grados de deterioro, ponderados en una escala de 0 a 13, fueron de 4-6 para los canalones, 1-3 para las losetas y 1-3 en el caso de las columnas, es decir que el grado de daño estuvo entre "despreciable" y "moderado". Los daños observados podrían haberse atenuado con un plan de mantenimiento sostenido en el tiempo que mantuviese la impermeabilidad de la cubierta.

Palabras clave: evaluación de patologías; hormigón pretensado; índice de daño; corrosión.

\section{INTRODUCCIÓN}


El término "pretensado" aparece por primera vez en 1929 en Alemania en la patente "método para la producción de acero reforzado en elementos de hormigón pretensado con barras rectas de refuerzo" desarrollada por Eugène Freyssinet (Llorente Zurdo, 2016). Posteriormente, el hormigón pretensado extendió su uso en todo tipo de estructuras como puentes de grandes luces, cubiertas industriales, depósitos de agua, durmientes de ferrocarril, viguetas, postes, etc (ACI 222.2R, 214). Por otra parte, las patologías que pueden presentar las estructuras de hormigón pretensado son variadas, no obstante, al igual que en toda estructura de hormigón, la manifestación más corriente de las mismas es a través de la aparición de fisuras. Esto ha dado lugar a numerosos estudios acerca de la fisuración del hormigón pretensado (Karayannis \& Chlioris, 2013; Dai et. al., 2016; Tong, 2016). Una particularidad es que la acción del pretensado tiende a mantener a las fisuras cerradas. Esto dificulta las inspecciones visuales de la estructura como un primer diagnóstico general el estado de la misma (Ercolani et. al., 2017).

Es importante destacar que el American Concrete Institute ha redactado un documento que brinda información general sobre la evaluación del daño por corrosión con recomendaciones sobre la reparación de cables monocordon no adherentes (ACI 423.4R, 1998), y no ha avanzado hacia los cables con adherencia. Este último tipo de estructura es más compleja de reparar al estar el acero embebido en el hormigón, y no se lo puede reemplazar en caso de un avanzado grado de corrosión, y las especificaciones del ACI no contemplan para este caso métodos de reparación (ACI 222.2R, 2014).

Cuando se debe realizar la evaluación de una estructura dañada es necesario elaborar un plan de trabajo, y en muchos casos recurrir a guías para organizar el mismo. Entre estas guía o métodos se encuentran la guía del Instituto Valenciano de la Edificación (IVE) (Guía IVE, 2005), el manual Contecvect (Contecvect, 1989), el manual DURAR (DURAR, 2000), el programa de evaluación simplificada basado en el DURAR (Rojas Moya, 2008), entre otros. Las guías de evaluación y rehabilitación para estructuras de hormigón armado sugieren en general tres etapas:

1) Inspección y evaluación preliminar: el objetivo es un reconocimiento visual (con pruebas y ensayos de un muestreo mínimo), obtener una primera estimación de los daños y un informe que permita la decisión sobre posibles actuaciones o intervenciones. 2) Inspección y evaluación complementaria: realización de inspecciones, pruebas y ensayos complementarios (profundidad de carbonatación, contenido de cloruros, resistividad, densidad de corriente, potencial de corrosión, ensayos semi o no destructivos, etc.), de forma más completa. El objetivo es obtener una mejor estimación de los daños (tanto de origen asociado a la durabilidad de los materiales como estructural) que determine con la mejor precisión posible las intervenciones a realizar. 3) Planificación de la intervención: se deciden los distintos modos de intervención más adecuados para la rehabilitación de la estructura a fin de restablecer el grado de seguridad y prolongar su vida útil (Helene y Pereira, 2007).

El objetivo del presente trabajo es la evaluación visual para determinar el grado de daño de los elementos pretensados/premoldeados que componen una planta industrial productora de cerámicos. De acuerdo al rango o grado de daño establecido en base a una escala de valores numéricos se diseñará un plan de evaluación más extensa, de reparación y mantenimiento, de forma que se verifique la seguridad y estabilidad de la estructura.

\section{DESCRIPCION Y ANTEDECEDENTES DE LA ESTRUCTURA}

La estructura se encuentra en la ciudad de Olavarría (Buenos Aires - Argentina) y está compuesto por cuatro edificios de una sola planta (Figura 1). La superficie total a relevar fue de $59.000 \mathrm{~m}^{2}$. La cubierta de todos los edificios está constituida por canalones y losetas huecas de hormigón pretensado, y sostenida por columnas premoldeadas (Figuras 2 y 3). Todos estos elementos 
fueron prefabricados y el relevamiento visual comprendió 448 canalones, 7000 losetas y 720 columnas.

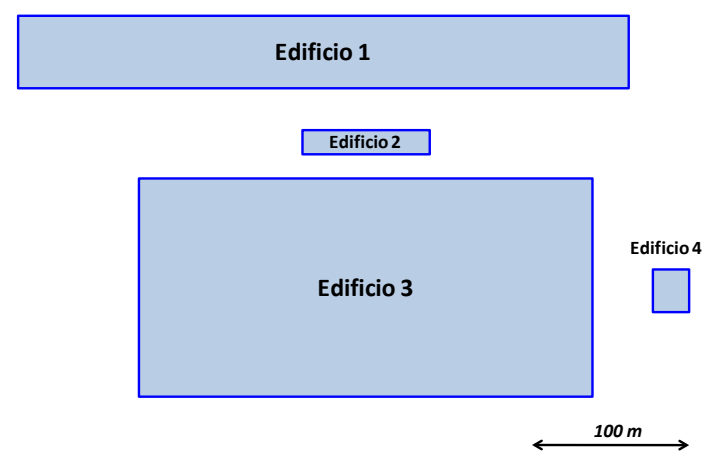

Figura 1. Plano de disposición en planta de los edificios relevados.

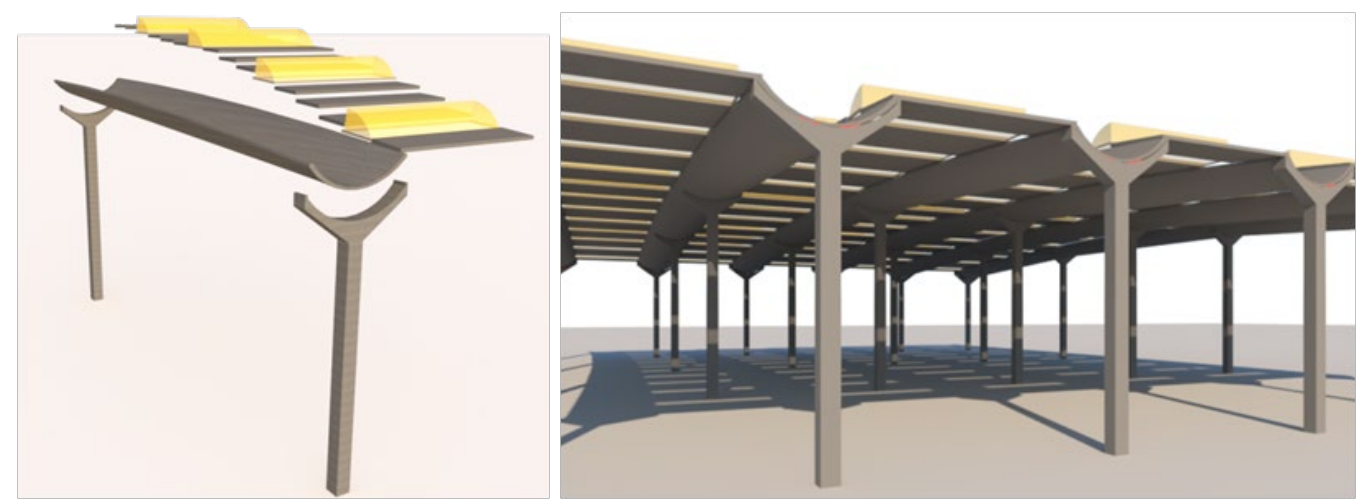

Figura 2. Columnas, canalones, losetas y lucernarios. Figura 3. Vista de un sector de la planta.

Las columnas están empotradas sobre fundaciones directas realizadas in-situ, tienen una sección rectangular de $40 \mathrm{~cm}$ x $45 \mathrm{~cm}$ y una altura variable entre 7 y $9 \mathrm{~m}$. En la parte superior tienen forma de "Y", abriéndose en dos "alas", cuyo borde superior sigue la curvatura de la sección transversal del canalón. Los canalones actúan como vigas y son una lámina de hormigón con forma de un paraboloide hiperbólico. Son elementos simplemente apoyados que descargan sobre columnas premoldeadas. La ventaja de su empleo se basa en la posibilidad de cubrir grandes luces con espesores mínimos de hormigón, por su rigidez debida a la doble curvatura y al efecto del pretensado. Los canalones tienen una longitud de $16,20 \mathrm{~m}$ y un espesor variable que va desde $7 \mathrm{~cm}$ en el centro de la luz hasta $12 \mathrm{~cm}$ en los extremos. El ancho de la sección en planta es de $2,70 \mathrm{~m}$ y la curvatura longitudinal tiene un radio de $\approx 180 \mathrm{~m}$. El peso de cada uno de ellos es de 11,56 toneladas. Las losetas terminan de cerrar la cubierta apoyándose en los bordes de los canalones y sus dimensiones son de $4 \mathrm{~m}$ por $1 \mathrm{~m}$, con un peso de $\approx 1$ tonelada. Por último, la cubierta se completa con los lucernarios de material traslucido, que dejan pasar la luz solar hacia el interior y están apoyados en los bordes de los canalones. Los edificios se fueron construyendo por sectores, el primer sector se construyó en el año 1974 y el último en 1992 (Tabla 1). Se puede observar que varios sectores de la planta están próximo cumplir los 50 años establecidos por el Reglamento CIRSOC 201:05 como vida útil de una estructura, con el mantenimiento previsto en el proyecto (CIRSOC 201:05). En este caso el mantenimiento no ha sido constante ni programado, siendo razonable que parte de las estructuras tengan algún grado de deterioro.

Tabla 1. Áreas y etapas de construcción de la planta industrial. 


\begin{tabular}{|c|c|c|c|}
\hline $\begin{array}{c}\text { Etapa de } \\
\text { construcción }\end{array}$ & $\begin{array}{c}\text { Área } \\
\text { cubierta }\left(\mathbf{m}^{2}\right)\end{array}$ & $\begin{array}{c}\text { Año de } \\
\text { construcción }\end{array}$ & Observaciones \\
\hline 1 & 14800 & 1974 & \multirow{4}{*}{$\begin{array}{l}\text { Sectores donde se había } \\
\text { colocado una membrana para } \\
\text { la aislación hidrófuga de la } \\
\text { cubierta. }\end{array}$} \\
\hline 2 & 8000 & $1974-78$ & \\
\hline 3 & 13500 & 1974-78 & \\
\hline 5 & 5500 & $1974-78$ & \\
\hline 6 & 12000 & 1982 & \multirow{2}{*}{$\begin{array}{l}\text { Desde su construcción no se } \\
\text { empleó aislación. }\end{array}$} \\
\hline 7 & 5200 & $1992-93$ & \\
\hline
\end{tabular}

La planta siempre tuvo el mismo uso industrial, sin embargo las líneas de producción fueron cambiadas en el transcurso del tiempo, tanto en su ubicación como en la tecnología empleada. Entonces, es posible que en etapas anteriores algunos sectores hayan estado sometidos a un ambiente más agresivo que el actual por el proceso de fabricación de cerámicos (p.e., temperaturas más elevadas, tipo de gases liberados, vibraciones, etc.).

\subsection{Antecedentes previos de las manifestaciones patológicas}

El principal inconveniente que presento la estructura fue la ruptura y caída de uno de los canalones, después de 41 años de servicio. Este evento llevo a prestar mayor atención en cuanto al estado de la cubierta de las plantas, y en el año 2015 se realizó una inspección para analizar las causas de la rotura y caída de un canalón. Se concluyo que el colapso fue debido a la corrosión de las armaduras de pretensado. Posteriormente, se realizó un estudio empleando la tomografía de hormigón armado y determinar las condiciones de los cables pretensados de seis canalones (Mariscotti et al, 2009). Se comprobó la falta de homogeneidad del hormigón y en solo uno de ellos tenía un cordón con la sección transversal reducida por corrosión. Dos meses más tarde, se realizaron inspecciones locales picando el hormigón de dos canalones para verificar el estado de los cordones pretensado. Se observó la cantidad de nervios, la presencia de óxido, el grado de corrosión en los mismos y, la reducción de la sección. Luego, a principios del año 2019, se comenzó a realizar la aislación hidrófuga de toda la cubierta, aplicando una membrana líquida impermeabilizante con poliuretano sobre cada canalón.

\section{METODOLOGÍA DE TRABAJO}

Ante los antecedentes descriptos, en el año 2019 la empresa decidió realizar un relevamiento visual preliminar para cuantificar la magnitud del problema de corrosión de toda la planta. La inspección visual, el ensayo no destructivo más antiguo, se efectúa en primera instancia y es la que permite tener más rápidamente una noción sobre las condiciones generales y particulares de la estructura (Di Maio, 2004). La información detallada de cada elemento, será de utilidad para determinar intervenciones sobre aquellos que presenten un grado de deterioro y en función de este, en qué momento realizarlas. También, este relevamiento será la base comparativa para futuros relevamientos de modo de establecer la evolución de los daños a través del tiempo. Se tomo como base para este relevamiento el procedimiento de la etapa de inspección y evaluación preliminar del IVE, con modificaciones para ajustarla al planteo del comitente (Figura 4). 


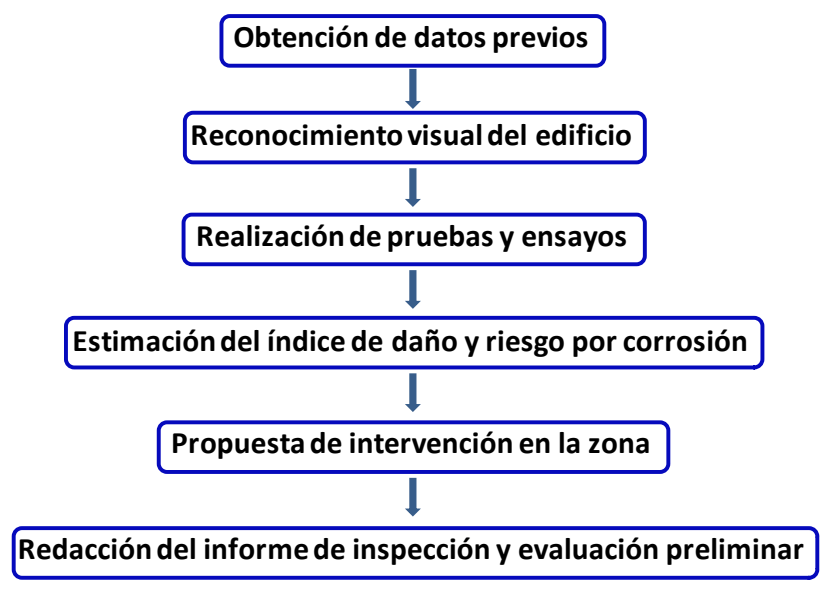

Figura 4. Esquema de la inspección y evaluación preliminar (Guía IVE, 2005).

La superficie a relevar se dividió en 21 zonas de inspección (Tabla 2). En todos los puntos la cubierta está expuesta a las mismas condiciones climáticas, aunque pueden variar las condiciones de exposición internas (temperatura, humedad ambiente, gases o agentes agresivos distintos, vibraciones, etc.). La identificación de cada elemento estructural se basa en un sistema de ejes ortogonales. En una dirección los ejes son alfabéticos y, en la otra, ejes numéricos. De este modo, la "Columna A7" es aquella que está en la intersección de los ejes A y 7, y en el caso del canalón "C3-WZ", su eje coincide con el eje 3 y se ubica entre los ejes W y Z.

Tabla 2. Designación y distribución de zonas de inspección.

\begin{tabular}{|c|c|c|c|c|c|}
\hline Zona & $\begin{array}{c}\text { Área de la zona } \\
\left(\boldsymbol{m}^{\mathbf{2}}\right)\end{array}$ & Zona & $\begin{array}{c}\text { Área de la zona } \\
\left(\boldsymbol{m}^{\mathbf{2}}\right)\end{array}$ & Zona & $\begin{array}{c}\text { Área de la zona } \\
\left(\boldsymbol{m}^{\mathbf{2}}\right)\end{array}$ \\
\hline 1 & 3520 & 8 & 1400 & 15 & 3100 \\
\hline 2 & 3730 & 9 & 2460 & 16 & 3290 \\
\hline 3 & 4060 & 10 & 2830 & 17 & 3290 \\
\hline 4 & 3030 & 11 & 3080 & 18 & 3290 \\
\hline 5 & 3520 & 12 & 2800 & 19 & 2700 \\
\hline 6 & 3730 & 13 & 2150 & 20 & 1700 \\
\hline 7 & 3700 & 14 & 1220 & 21 & 400 \\
\hline
\end{tabular}

Para realizar la evaluación se diseñaron tres planillas diferentes una para cada elemento estructural a relevar (columnas, canalones y losetas). En las tres se indica la fecha en que se hizo el relevamiento y la zona o sector a la que pertenecen los elementos relevados. Cada planilla tiene el espacio para registrar: la identificación del elemento según el sistema de ejes, celdas para tildar aquellas lesiones que aparezcan (tipo, tamaño, forma y ubicación de las mismas), un esquema para dibujar las patologías, un espacio para describir cualquier observación que se considere necesaria, y otro para identificar el número de fotografía correspondiente a lesión observada. En las Figuras 5, 6 y 7 se muestran los modelos de las planillas, en forma simplificada. Los principales elementos utilizados en campo fueron binoculares, cámara digital con zoom, planillas y un elevador para aquellos elementos que presentaban mayor índice de riesgo se llevó a cabo una inspección más detallada de observando más de cerca la superficie dañada. 


\begin{tabular}{|l|l|l|}
\hline ZONA No & \multicolumn{2}{|c|}{ FECHA: } \\
\begin{tabular}{|l|l|l|}
\hline Nombre & Fotos $\mathbf{N}^{\mathbf{0}}$ & Observaciones \\
\hline Columna: & & \\
\hline Columna: & & \\
\hline & & \\
\hline
\end{tabular}
\end{tabular}

Figura 5. Planilla para el relevamiento de columnas.

\begin{tabular}{|c|c|c|c|c|c|c|c|c|c|c|c|c|}
\hline \multicolumn{8}{|l|}{ ZONA: } & \multicolumn{5}{|c|}{ FECHA: } \\
\hline \multirow[t]{2}{*}{ Nombre } & \multicolumn{7}{|c|}{ Manchas y filtaciones } & \multicolumn{5}{|c|}{ Fisuras } \\
\hline & Long. & Óxido & Eflor. & Humedad & Tamaño & Cantidad & Ubicación & Transv. & Long. & Paralela & 2450 & Óxido \\
\hline & & & & & & & & & & & & \\
\hline & & & & & & & & & & & & \\
\hline \multirow{4}{*}{ 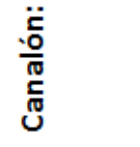 } & & & & & & & & & & & & \\
\hline & & & & & & & & & & & & \\
\hline & & & & & & & & & & & & \\
\hline & & & & & & & & & & & & \\
\hline & & & & & & & & & & & & \\
\hline & & & & & & & & & & & & \\
\hline \multirow{5}{*}{ 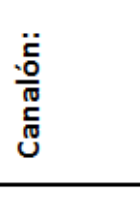 } & & & & & & & & & & & & \\
\hline & & & & & & & & & & & & \\
\hline & & & & & & & & & & & & \\
\hline & & & & & & & & & & & & \\
\hline & & & & & & & & & & & & \\
\hline
\end{tabular}

Figura 6. Planilla para el relevamiento de canalones (vista parcial).

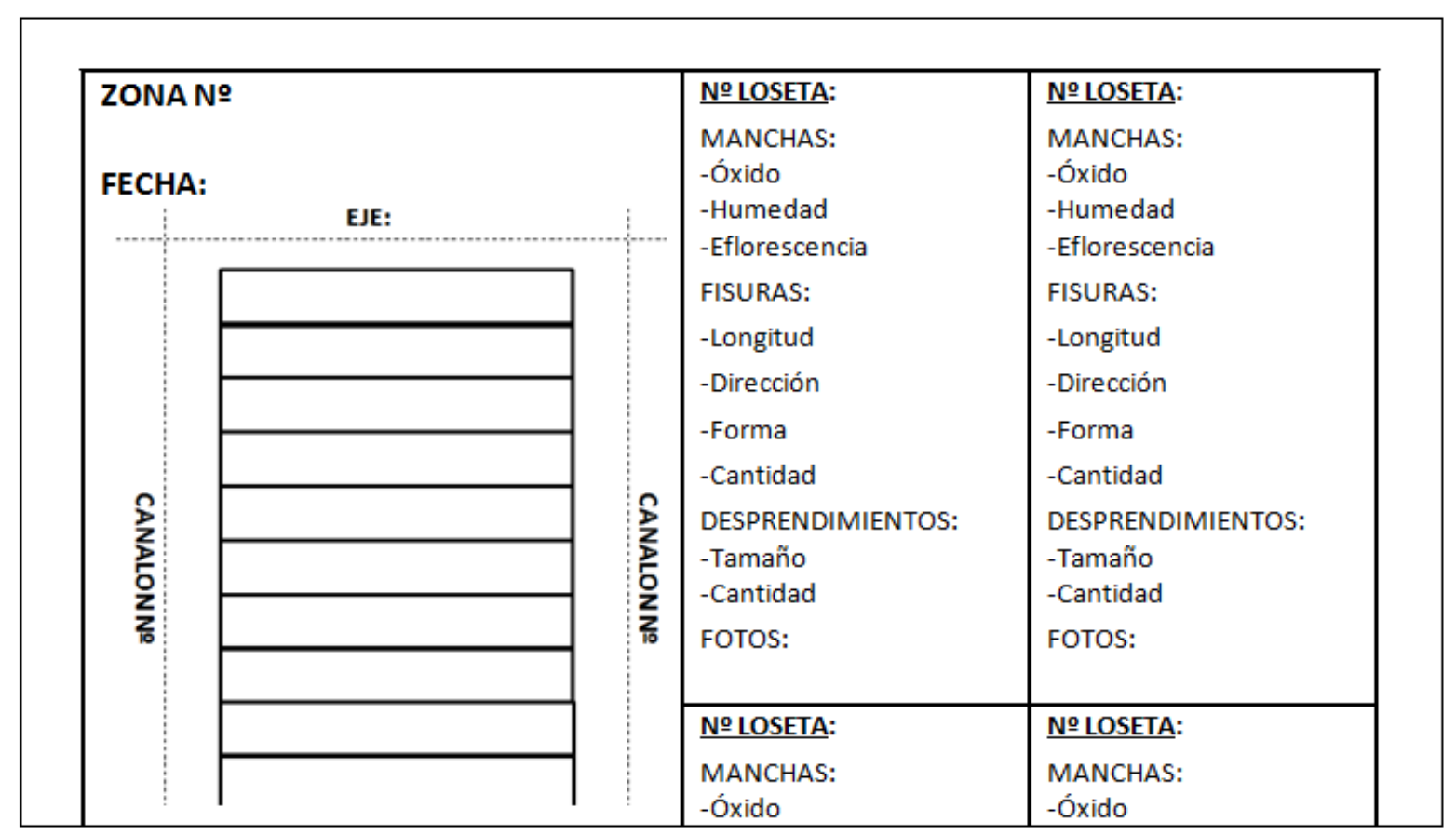

Figura 7. Planilla para el relevamiento de losetas huecas. 
En esta etapa preliminar no se realizaron ensayos físicos, químicos, mecánicos o no destructivos. El criterio fue determinar en forma aproximada (visualmente) el grado de deterioro y tipo de síntomas que presentaban los elementos estructurales. En base a esta información, se establecerá el tipo de ensayos complementarios que convendrá aplicar. Posteriormente se definirán las estrategias de intervención por cuestiones de seguridad, los tiempos para las diferentes reparaciones, y el seguimiento del daño en determinados elementos estructurales críticos.

\subsection{Estimación del índice de daño/evaluación del estado de la estructura}

Si bien se han establecido varias metodologías para la evaluación del daño de una estructuras (Guía IVE, 2005; Contecvect, 1989; DURAR, 2000 y Rojas Moya, 2008) se decidió establecer una calificación en función de un "índice de daño" basado en los datos obtenidos en la inspección visual, ponderando diversos factores (como se detallan en las planillas). Para ello se estableció una escala en función del grado de deterioro que se observaba. Si bien la escala es relativamente arbitraria, la misma permite distinguir zonas con cierto grado de deterioro de otras con mayor o menor daño. Después de este relevamiento preliminar, de acuerdo con los resultados se pasará a otra etapa donde se realizarán otros ensayos complementarios y se definirán las intervenciones.

Se diseño una escala del índice de daño para cada elemento en base a los valores establecidos en la Tabla 3, que se asignan de acuerdo a los síntomas observados. Cuando en un mismo canalón se observan más de un síntoma distinto, se establece como valor de índice de daño al mayor valor entre ellos.

Tabla 3. Índice de daño empleado para calificar el deterioro de los canalones.

\begin{tabular}{|c|c|c|}
\hline Síntomas & $\begin{array}{l}\text { Índice de } \\
\text { Daño }\end{array}$ & Observaciones \\
\hline No se observan & 0 & \\
\hline $\begin{array}{l}\text { Manchas de filtraciones que ingresa en } \\
\text { unión entre lucernarios y/o losetas }\end{array}$ & 0 & \\
\hline Manchas de eflorescencia & 1 & \\
\hline Material poroso & 1 & $\begin{array}{l}\text { Si se observan poros de mayor tamaño o cantidad se } \\
\text { aumenta un número más el grado de daño. }\end{array}$ \\
\hline $\begin{array}{l}\text { Filtraciones puntuales (no proveniente } \\
\text { de las uniones de elementos) a través } \\
\text { de fisuras, poros del material }\end{array}$ & 5 & $\begin{array}{l}\text { Cuando son filtraciones a través de fisuras, se } \\
\text { aumenta un número más el grado de daño. }\end{array}$ \\
\hline Manchas de óxido & $1-6$ & $\begin{array}{l}\text { El valor del grado de daño varía en función de la } \\
\text { concentración y cantidad de las manchas de óxido. }\end{array}$ \\
\hline Fisuras inclinadas a $45^{\circ}$ & 6 & \multirow{5}{*}{$\begin{array}{l}\text { Si son varios/as de esa longitud se debe aumentar } \\
\text { un número más al grado de daño. }\end{array}$} \\
\hline $\begin{array}{l}\text { Fisuras longitudinales: } \\
<\text { a } 50 \mathrm{~cm} \\
\approx 100 \mathrm{~cm} \text { o más } \\
\text { Toda la longitud del canalón }\end{array}$ & $\begin{array}{l}4 \\
5 \\
6\end{array}$ & \\
\hline $\begin{array}{l}\text { Fisuras transversales: } \\
<\text { a } 50 \mathrm{~cm} \\
\approx 100 \mathrm{~cm} \\
\text { Todo el ancho del canalón }\end{array}$ & $\begin{array}{l}4 \\
6 \\
7\end{array}$ & \\
\hline $\begin{array}{l}\text { Desprendimientos longitudinales: } \\
<\text { a } 50 \mathrm{~cm} \\
\approx 100 \mathrm{~cm} \\
\text { Toda la longitud del canalón }\end{array}$ & $\begin{array}{c}8 \\
9 \\
10 \\
\end{array}$ & \\
\hline $\begin{array}{l}\text { Desprendimientos transversales: } \\
<\text { a } 50 \mathrm{~cm} \\
\approx 100 \mathrm{~cm} \\
\text { Todo el ancho del canalón }\end{array}$ & $\begin{array}{c}8 \\
9 \\
10\end{array}$ & \\
\hline
\end{tabular}




\begin{tabular}{|l|c|l|}
\hline Armadura expuesta & 10 & $\begin{array}{l}\text { Se aplica tanto al caso de desprendimientos del } \\
\text { recubrimiento donde la armadura queda a la vista, } \\
\text { como también, cuando los canalones presentan } \\
\text { errores de ejecución quedando la armadura fuera del } \\
\text { material por la ausencia de separadores. }\end{array}$ \\
\hline $\begin{array}{l}\text { Desprendimientos mayores a los } \\
\text { anteriores }\end{array}$ & $11-13$ & \\
\hline Rotura del canalón & $\begin{array}{l}\text { Intervenir } \\
\text { enseguida }\end{array}$ & \\
\hline Otro daño importante & & \\
\hline
\end{tabular}

\section{RESULTADOS Y SU DISCUSION}

\subsection{Evaluación de los canalones}

Empleando la metodología descripta anteriormente se informan los resultados obtenidos en la evaluación preliminar de los canalones. Como ejemplo del método de relevamiento se informa solo una zona de la planta y en ella la mayor parte de los canalones presentan un nivel de deterioro intermedio (generalmente manchas de óxido, fisuras y filtraciones), y solo 6 presentan un daño alto. En la Figura 8 se indica la distribución, en porcentaje, de las patologías observadas en los canalones.

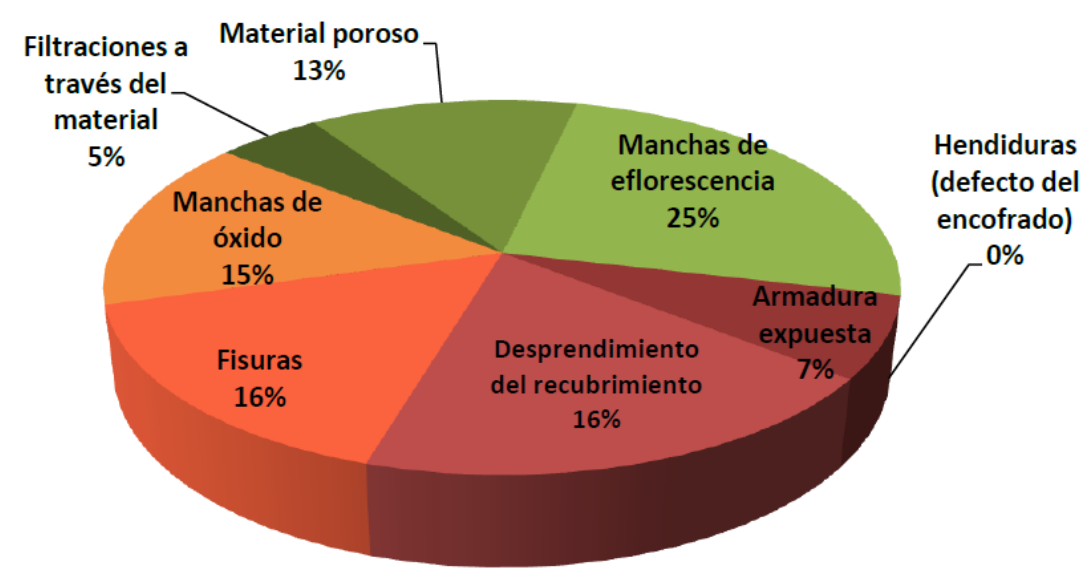

Figura 8. Distribución relativa de las patologías observadas en los canalones de la zona elegida.

A modo de resumen para evidenciar la distribución del grado de deterioro y el nivel de riesgo que presenta cada canalón de la cubierta, se elaboró una escala colorimétrica para pintar los canalones en el plano correspondiente. Esto permite reconocer en forma rápida y sencilla cuales son los más afectados, aunque se elaboraron informes técnicos con sus respectivos anexos con un minucioso grado de detalle. Además, esta escala permite tener una visión global del estado de la cubierta como síntesis de los informes. En la Figura 9 se observa la imagen resultante de una de las zonas relevadas en la que se puede observar los distintos niveles de daño en cada canalón de acuerdo con la escala colorimétrica adoptada.

Una vez finalizado el trabajo de relevamiento se calculó el porcentaje de canalones con cada rango de daño para cada zona, y para el total de la planta. Estos valores estiman la extensión en la que se deberá aplicar cada tipo de intervención. En la Tabla 4 se indican las cantidades de canalones con diferentes grados de daño correspondientes a la zona de la Figura 9. 

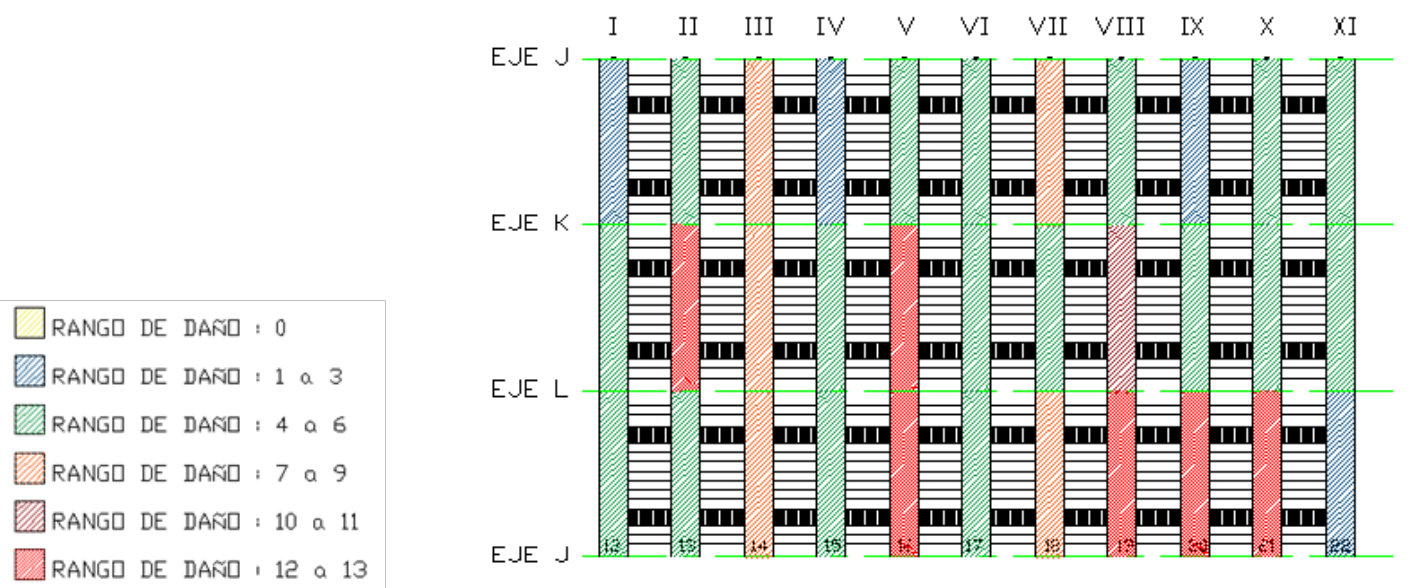

Figura 9. Imagen de una de las zonas relevadas donde se indica el rango de daño de cada canalón con escala colorimétrica.

Tabla 4. Cantidad de canalones de la zona de la Figura 9 con distintos grados de daño.

\begin{tabular}{|l|c|c|c|}
\hline Color & Rango de daño & Cantidad de canalones & Cantidad en \% \\
\hline Negro & No fueron relevado & 0 & 0,0 \\
\hline Amarillo & 0 & 0 & 0,0 \\
\hline Azul & $1-3$ & 4 & 12,1 \\
\hline Verde & $4-6$ & 17 & 51,5 \\
\hline Naranja & $7-9$ & 5 & 15,2 \\
\hline Bermellón & $10-11$ & 1 & 3,0 \\
\hline Rojo & $12-13$ & 6 & 18,2 \\
\hline
\end{tabular}

Luego de haber relevado visualmente la cubierta, se llevó a cabo una inspección más detallada utilizando un elevador de aquellos canalones que presentaban un mayor índice de riesgo con el objetivo de observar más de cerca la superficie dañada. De esta evaluación adicional se puede destacar:

- En algunos puntos donde la armadura pasiva estaba expuesta al observarla de cerca se constato que tenía una pérdida de sección importante debida al escaso espesor de recubrimiento (Figuras 10).

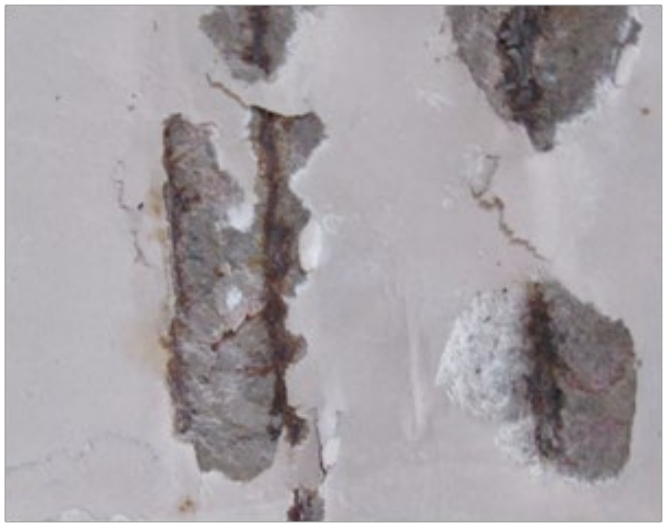

(a)

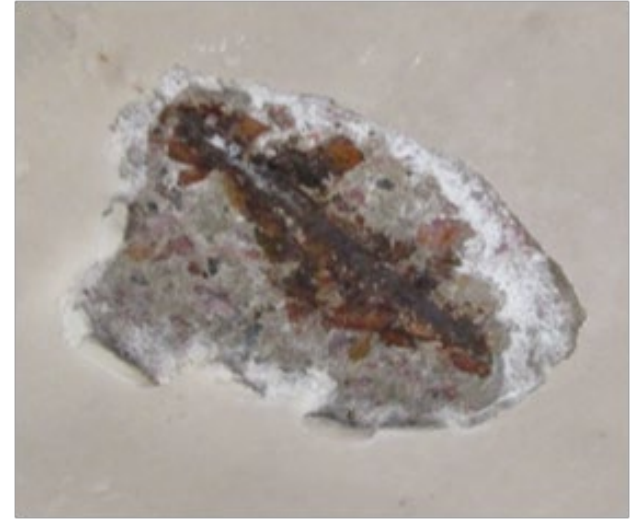

(b)

Figura 10. Imágenes de la superficie de algunos canalones que presentan desprendimientos del recubrimiento (a) y armaduras con corrosión (b). 
- En aquellos casos donde se observó parte del material de recubrimiento desprendiéndose o suelto al arrancarlo se advirtió que los cables de pretensado estaban corroídos, y se observo la existencia de oquedades debido a una alta densidad de armaduras y a defectos en la mezcla o en el proceso de vibrado del hormigón en estado fresco (Figuras 11).

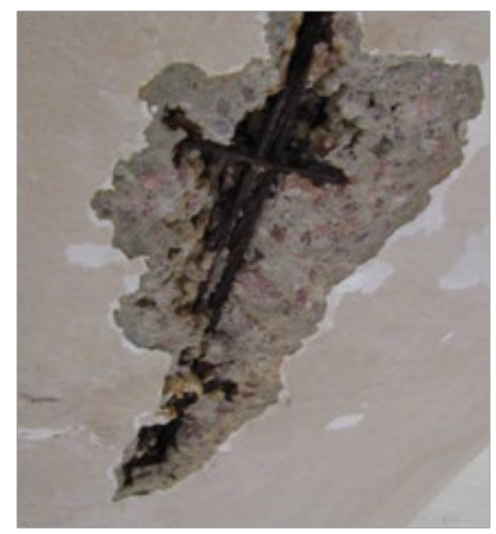

(a)

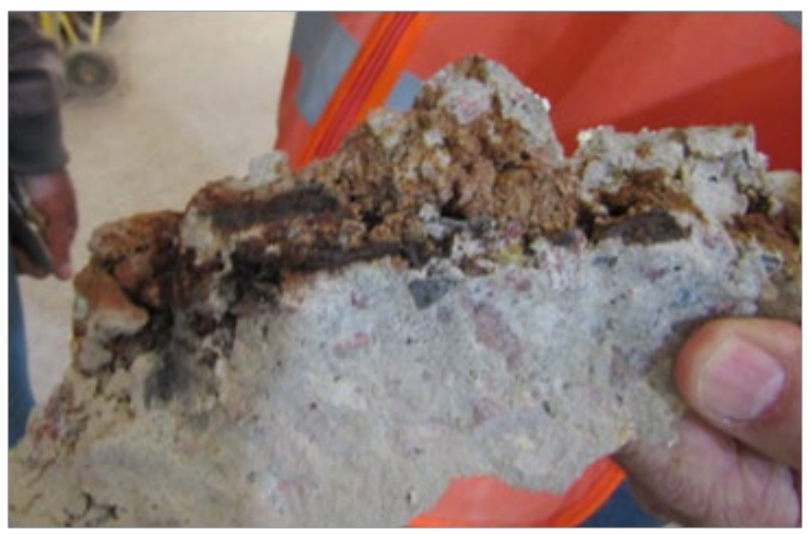

(b)

Figura 11. Imágenes de la superficie de algunos canalones que presentan desprendimientos del recubrimiento con cables de pretensado oxidados (a) y grandes oquedades (b).

\subsection{Evaluación de las losetas}

De la misma forma que para el estudio de los canalones se diseñó una escala de daño para las losetas en concordancia con la planilla de la Figura 7. La tabla es muy similar a la anterior, sin embargo, el tipo y nivel de incidencia de los síntomas son diferentes cuando ocurren en los canalones que cuando aparecen en las losetas o columnas por el impacto que tendría un posible colapso (el área de la cubierta de una loseta es de $4 \mathrm{~m}^{2}$, y el área de incidencia de un canalón con las viguetas que apoyan sobre él es de $136 \mathrm{~m}^{2}$ ). En la Figura 12 se indica la distribución, en porcentaje, de las patologías observadas en las losetas.

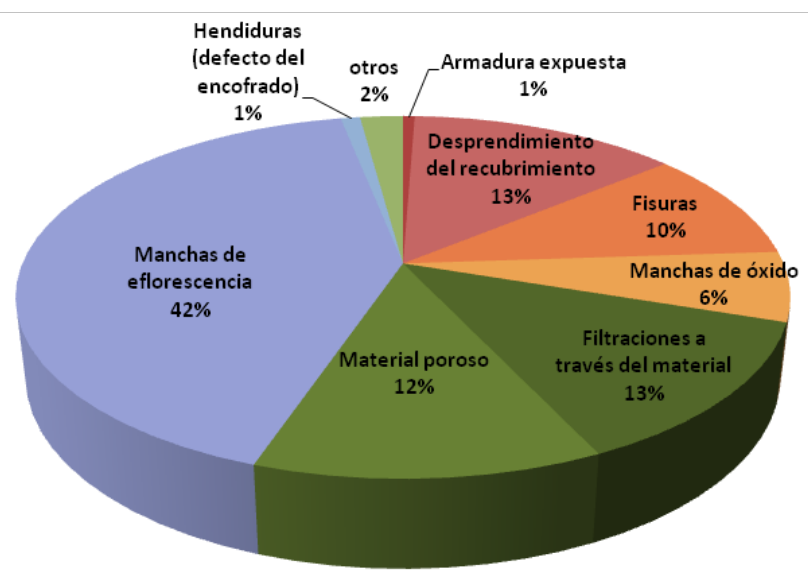

Figura 12. Distribución relativa de las patologías observadas en las losetas de la zona elegida.

En la Figura 13 se muestra la aplicación del índice de daño a losetas correspondientes a la misma zona de la plata industrial que se mostró para los canalones. Por otro lado, en la Tabla 5 se calculó el porcentaje de cada grado de daño observado en las losetas. 


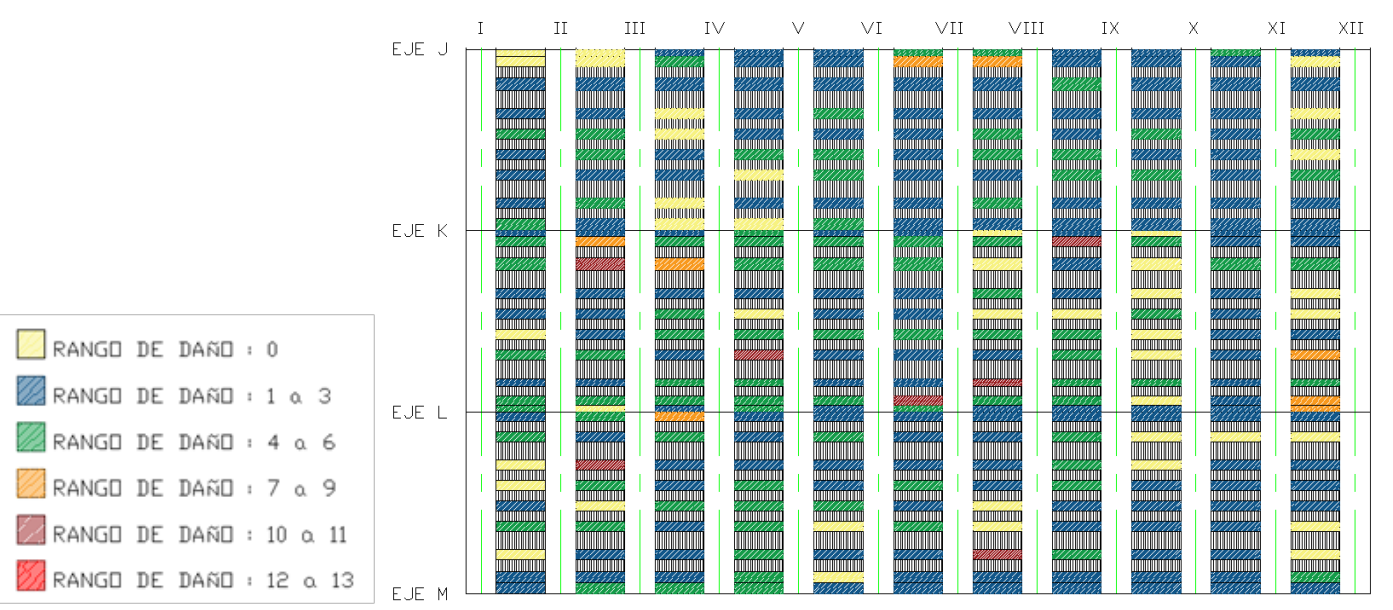

Figura 13. Imagen de una de las zonas relevadas donde se indica el rango de daño de cada loseta con escala colorimétrica.

Tabla 5. Cantidad de losetas de la zona de la Figura 13 con distintos grados de daño.

\begin{tabular}{|l|c|c|c|}
\hline Color & Rango de daño & Cantidad de losetas & Cantidad en \% \\
\hline Negro & No fueron relevadas & 0 & 0,0 \\
\hline Amarillo & 0 & 42 & 12,8 \\
\hline Azul & $1-3$ & 162 & 49,4 \\
\hline Verde & $4-6$ & 109 & 33,2 \\
\hline Naranja & $7-9$ & 8 & 2,4 \\
\hline Rojo & 10 & 7 & 2,2 \\
\hline
\end{tabular}

\subsection{Evaluación de las columnas}

De un modo similar a lo realizado con los canalones y las losetas, se diseño una escala de daño para las columnas premoldeadas. En la Figura 14 se indica la distribución, en porcentaje, de las patologías observadas en las columnas y en la Figura 15 se aplica la escala de daño al mismo sector de la planta. En la Tabla 6 se indican la cantidad de columnas con diferentes grados de daños.

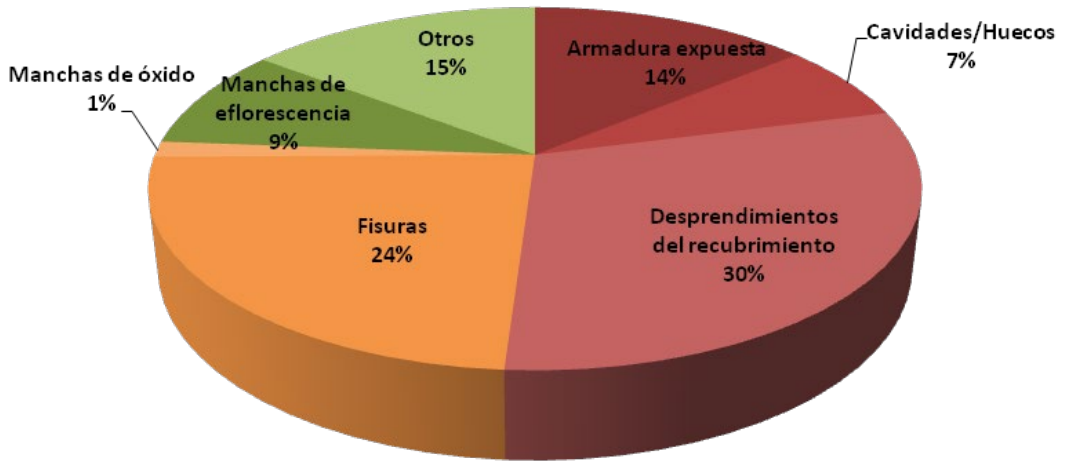

Figura 14. Distribución relativa de las patologías observadas en las columnas de la zona elegida. 


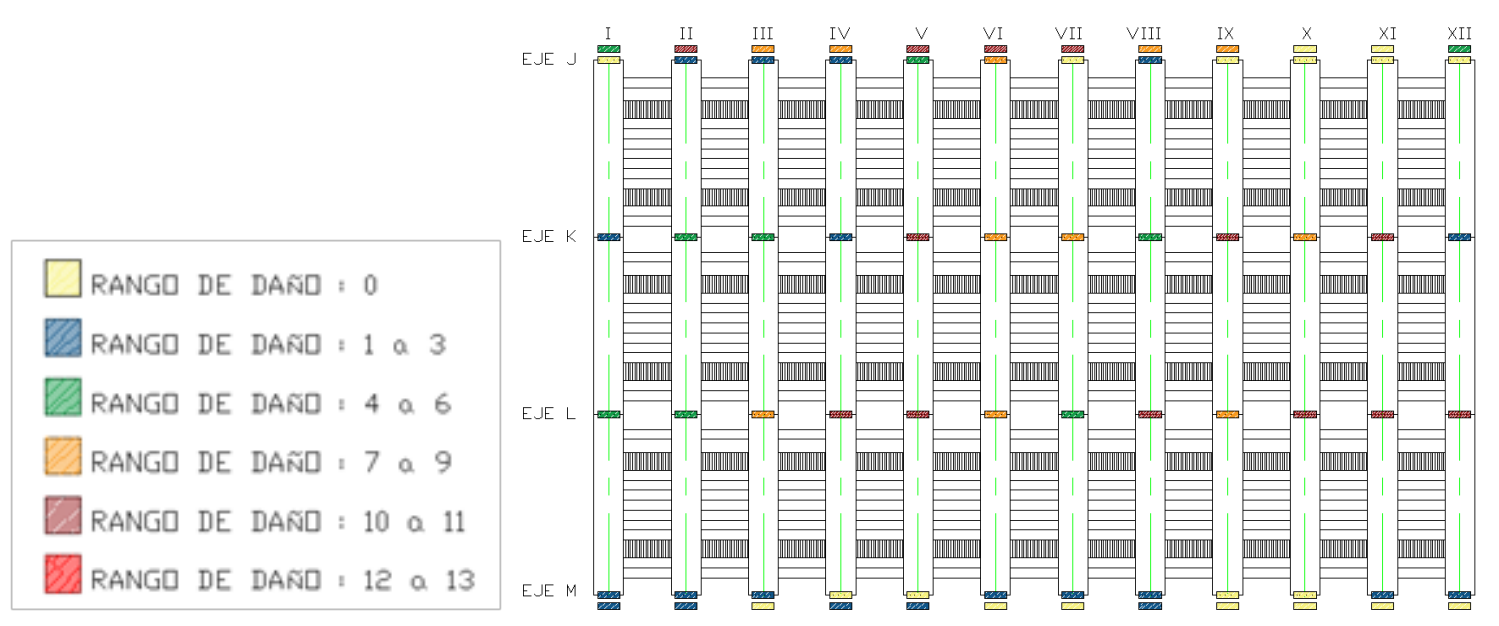

Figura 15. Imagen de una de las zonas relevadas donde se indica el rango de daño de cada columna con escala colorimétrica.

Tabla 6. Cantidad de columnas de la zona de la Figura 15 con distintos grados de daño.

\begin{tabular}{|l|c|c|c|}
\hline Color & Rango de daño & Cantidad de columnas & Cantidad en \% \\
\hline Negro & No fueron relevadas & 0 & 0,0 \\
\hline Amarillo & 0 & 19 & 26,4 \\
\hline Azul & $1-3$ & 20 & 27,8 \\
\hline Verde & $4-6$ & 9 & 12,5 \\
\hline Naranja & $7-9$ & 11 & 15,3 \\
\hline Rojo & 10 & 13 & 18 \\
\hline
\end{tabular}

\subsection{Propuesta de tiempos de intervención de la estructura}

De acuerdo con los métodos existentes sobre evaluaciones de estructuras que presentan patologías asociadas a la corrosión de las armaduras y teniendo en cuenta el alcance del relevamiento visual se estableció una clasificación de la urgencia de intervención (nueva inspección o reparación/refuerzo de la estructura). En base al índice de daño que presenta cada elemento, se establecen plazos de intervención o inspecciones futuras, de un modo similar al planteado en la bibliografía (Rojas Moya, 2008). Se agruparon los rangos de daño en cuatro grupos o escala de daño: Despreciable, Moderado, Severo y Muy severo. Luego, para cada uno de estos rangos de daño se estimaron los intervalos de tiempo aconsejables de intervención o reparación (Tabla 7).

Tabla 7. Tiempos propuestos para realizar la intervención según la escala de daño.

\begin{tabular}{|l|c|c|}
\hline \multicolumn{1}{|c|}{ Grado de Daño } & Índice de Daño & Urgencia de intervención (años) \\
\hline Despreciable & $0-2$ & $>6$ \\
\hline Moderado & $3-6$ & $3-6$ \\
\hline Severo & $7-9$ & $1-3$ \\
\hline Muy severo & $10-13$ & $0-1$ \\
\hline
\end{tabular}


El tipo de intervención dependerá del resultado obtenido en la evaluación:

- Para periodos $>6$ años, se recomienda una nueva "inspección preliminar" una vez pasado ese tiempo. En este caso, se deberá utilizar al presente estudio para determinar el avance del daño.

- Para periodos de 3 a 6 años, se recomienda una nueva "inspección preliminar" de la estructura dentro de ese período de tiempo (midiendo la velocidad de corrosión, en lo posible, o empleando métodos no destructivos u otros que permitan obtener información más fehaciente sobre el estado de los elementos estructurales).

- Para periodos entre 1 a 3 años, se recomienda una "evaluación complementaria", lo cual es una evaluación más detallada, realizar ensayos complementarios sobre el estado de las armaduras, etc. dentro de ese plazo.

- Para periodos $\leq 1$ año, se recomendaría una actuación rápida, como el apuntalamiento o resolución similar.

- Por ser un relevamiento visual, en los elementos estructurales más comprometidos se aconseja realizar ensayos (semi y no destructivos) y ensayos complementarios para tener mayor seguridad.

Finalmente, del relevamiento efectuado se desprende la importancia del mantenimiento de las estructuras de hormigón armado, tema que no es habitual de encontrar, y que representa una mayor vida útil y la conservación del grado de seguridad de la estructura. El mantenimiento de plantas/edificios de grandes superficies como en este caso $\left(59000 \mathrm{~m}^{2}\right)$ requiere de una programación sistemática en el tiempo, dado los costos que implica su realización, atendiendo el trabajo por zonas o por tipo de elementos estructurales.

Un plan de mantenimiento es esencial. Los canalones son láminas delgadas de muy poco espesor de recubrimiento y considerable área superficial expuesta (elevada relación $\mathrm{m}^{2} / \mathrm{m}^{3}$ ), lo cual lo hace muy susceptible frente al fenómeno de carbonatación. Por otro lado, a esta situación se le suma la presencia de cables pretensados que tienen una mayor susceptibilidad de corroerse. Cabe destacar, también, la importancia de la etapa de ejecución ya que, en el caso de los canalones, se encontraron oquedades y recubrimientos muy delgados que afectan a la resistencia y durabilidad del mismo. Pequeños errores de ejecución, pueden llevar a elevados costos de mantenimiento y rehabilitación.

\section{CONCLUSIONES}

El trabajo realizado permite efectuar las siguientes consideraciones a modo de conclusiones:

- El índice de daño es uno de los criterios a tomar en cuenta para establecer los tiempos para efectuar intervenciones en las distintas zonas de la estructura, y los tiempos de los próximos relevamientos para evaluar la evolución del estado de la planta.

- Si bien el relevamiento visual detecta el estado de los elementos estructurales y las diferencias en distintos grados de deterioro, siempre es aconsejable efectuar una siguiente etapa donde se empleen ensayos o técnicas que puedan indicar con mayor precisión el deterioro interno del material.

- El efecto de las filtraciones de agua, la falta de impermeabilización, la presencia de membranas deterioradas (membrana cuarteada que acumula el agua), la realización de huecos en columnas (dejando la armadura expuesta), amures en canalones y losetas (sumando cargas no previstas en elementos estructurales), sobre las estructuras de hormigón armado o pretensado constituyen el principal motivo del origen de las eflorescencias, fisuras, desprendimientos y colapsos debidos a la corrosión de las armaduras. 
- Los principales elementos que conforman la cubierta (canalones) son relativamente pesados, complejos en su geometría, y por tener al acero de pretensado con adherencia resultan complejos de rehabilitar.

\section{REFERENCIAS}

- American Concrete Institute. (2014) ACI 222.2R-14: "Corrosion of Prestressing Steels", Farmington Hills, MI, USA.

- American Concrete Institute. (2014) ACI 423.4R-14: “Corrosión y reparación de cables monocordón no adherentes”, Farmington Hills, MI, USA.

- Dai, L., Wang, L., Zhang, J. and Zhang, H. (2016) "A global model for corrosion-induced cracking in prestressed concrete structures”, Engineering Failure Analysis. 62:263-275.

- Di Maio, A. (2004) "Técnicas y ensayos de evaluación de estructuras afectadas por patologías" Jornadas de Patología de Estructuras de Hormigón Armado, La Plata, Argentina.

- DURAR (2000) "Manual de Inspección, Evaluación y Diagnóstico de Corrosión en Estructuras de Hormigón Armado", Ed. CYTED-Programa Iberoamericano de Ciencia y Tecnología para el Desarrollo, España.

- Ercolani, G.D., Ortega, N.F. y Félix, D.H. (2017) "Detección de fallas en vigas de estructuras pretensadas mediante técnicas estáticas”, CONPAT 2017, Asunción, Paraguay.

- Geocisa, CONTECVET. (2002) "Manual de evaluación de estructuras afectadas por corrosión de la armadura" Madrid, España.

- Helene, P. R. y Pereira, F. (Eds). (2007) "Rehabilitación y mantenimiento de estructuras de concreto", ISBN 85-60457-00-3, San Pablo, Brasil, p.598.

- Instituto Valenciano de la Edificación. (2005) "Guía para la Inspección y Evaluación Preliminar de estructuras de hormigón en edificios existentes”. Valencia, España, p.114.

- Karayannis, C.G. and Chlioris, C. E. (2013) "Design of partially prestressed concrete beams based on the cracking control provisions", Engineering Structures. 48:402-416.

- Llorente Zurdo, M. P., (2016), "El pretensado: la disolución de las tipologías constructivas en la arquitectura del $S X X$ ”, Tesis Doctoral, Universidad Politécnica de Madrid, p. 638.

- Mariscotti, M. A., Jalinoos, F., Frigerio, T., Ruffolo, M. and Thieberger, P. (2009) "GammaRay Imaging for Void and Corrosion Assessment”, ACI International. 31(11): pp. 48-53.

- Peralta, M. H. y Ercoli, N.L. (2005) "Measuring the dynamic properties to detect structural damage” - IV International ACI/ CANMET. Conference of Quality of Concrete Structures and Recent Advances in Concrete Materials and Testing, Olinda, Brazil, pp. 463-475, ISBN 85-98576-08-5.

- Reglamento CIRSOC 201. (2005) “Reglamento Argentino de Estructuras de Hormigón”, Ed. INTI, Argentina.

- Rojas Moya, G. (2008) “Evaluación de Estructuras de Concreto por Corrosión”, Tecnología en Marcha. 21 (4):69-78.

- Tong, T., Liu, Z., Zhang, J. and Yu, Q. (2016) "Long-term performance of prestressed concrete bridges under the intertwined effects of concrete damage, static creep and trafficinduced cyclic creep", Engineering Structures. 127:510-524. 